\title{
Massive reversible pulmonary cysts in a patient with AIDS
}

\author{
Julio Collazos, Eduardo Martínez, Jose Mayo, Jose Luis Miguélez
}

\begin{abstract}
Summary
We report the case of a patient with AIDS who presented with bilateral, massive pulmonary cystic disease and pneumothorax presumably caused by Pneumocystis carinii which responded to treatment with cotrimoxazole. Follow-up with sequential chest $X$-rays and computed tomography scans showed a progressive improvement in the lung cysts which had resolved almost completely 11 months later. Several theories have been proposed to explain the cavitary lesions in this infection. The course observed in our patient suggests that a non-destructive mechanism was responsible for his lung cysts.
\end{abstract}

Keywords: AIDS, pulmonary cysts, Pneumocystis carinii, pneumothorax

Cavitary lung disease is a well-known form of presentation of Pneumocystis carinii pneumonia in AIDs patients. ${ }^{1-4}$ These patients usually have small cavities within lung infiltrates, although larger ones have also been reported., ${ }^{2,5}$ Herein we report the case of a patient who developed bilateral, massive cavitary lung disease presumably caused by $P$ carinii which had resolved almost completely 11 months later.

\section{Case report}

A 32-year-old HIV-positive man was admitted with an eight-month history of malaise, weight loss, and low-grade fever, and a history of chest pain and dyspnea since the previous day. He had been an intravenous drug abuser. He was receiving pyrimethamine sulfadoxine as prophylaxis for $P$ carinii pneumonia. Leucocyte count was $2.5 \times 10^{9} / 1, \mathrm{CD} 4$ cell count $0.012 \times 10^{9} / 1$, haemoglobin $11 \mathrm{~g} / \mathrm{dl}$, and erythrocyte sedimentation rate $122 \mathrm{~mm} / \mathrm{h}$. Right pneumothorax was evident on chest X-ray, along with many lung cysts. Another chest $\mathrm{X}$-ray and computed tomography (CT) scan, obtained after resolution of the pneumothorax with a chest tube, showed extensive cystic lung disease involving both lungs (figures 1 and 2). Direct staining of sputum, bronchoaspirate and bronchoalveolar lavage (BAL) fluid for mycobacteria, fungi, viruses and $P$ carinii were negative. Blood, BAL fluid, bronchoaspirate material, and several sputum cultures were negative for bacteria, mycobacteria, and fungi. Serology for toxoplasma and cytomegalovirus were negative. The patient was treated with cotrimoxazole for 21 days with rapid improvement and was discharged with a diagnosis of $P$ carinii pneumonia with cotrimoxazole as secondary prophylaxis. A chest X-ray and CT scan obtained six months later showed marked improvement of the cystic disease. The patient was admitted again 11 months after the previous admission due to generalised Cryptococcus neoformans infection which responded to fluconazole. Both chest X-ray and CT scan revealed a marked regression of the cysts in both lungs (figures 1 and 2). Four months after the last admission the patient remains well.

\section{Discussion}

Although we failed to recover any microorganisms, we believe that $P$ carinii is the most probable cause of this disease. This agent has

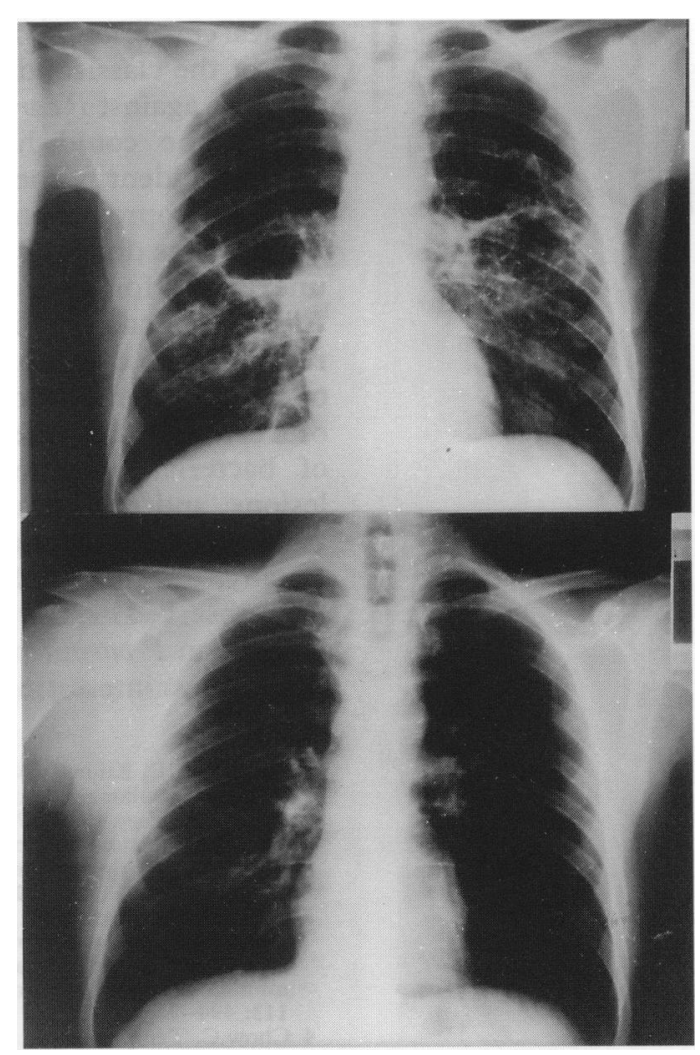

Figure 1 Chest X-ray obtained during the first admission (top) showing massive cystic disease in both lungs with an air-fluid level in a large cyst. Eleven months later the cysts had resolved almost completely (bottom) 


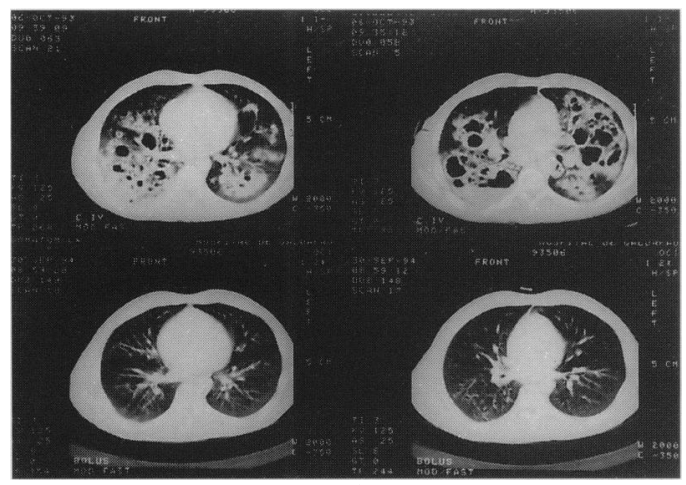

Figure 2 CT scans obtained during the first admission (top) and 11 months later (bottom) showing remarkable improvement of the lesions

been associated with pneumatocele and cyst formation in $7-34 \%$ of cases in AIDS patients, ${ }^{4-6}$ and pneumothorax and pneumomediastinum are well-known complications of infection. ${ }^{2,7}$

The strongest arguments against $P$ carinii are the long duration of symptoms before the patient received effective antipneumocystis therapy and the absence of identification of the parasite in respiratory secretions. However, three patients have recently been published who developed a chronic form of the disease lasting for up to two years with a stable clinical course. $^{8}$ Interestingly, $P$ carinii was not identified in respiratory secretions, BAL fluid and transbronchial biopsy, and all required thoracoscopy and biopsy for diagnosis. The authors suggested that a productive or destructive form exists which follows a prolonged clinical course as opposed to the acute presentation of the classical disease. ${ }^{8}$ In addition, prophylaxis against $P$ carinii with pyrimethaminesulfadoxine could have contributed to the failure to identify the pathogen.

The improvement with cotrimoxazole therapy was dramatic, which would have been unexpected for other opportunistic pathogens. Although a bacterial infection might also have responded to cotrimoxazole, the long duration of the symptoms, the mild course in the absence of treatment, the absence of previous episodes of bacterial pneumonia, the extent of the lesions, and the failure to recover a bacterial pathogen argue strongly against this aetiology, Finally, the sum of these alterations have not been observed in AIDS patients with infections other than $P$ carinii pneumonia. ${ }^{9}$

The most interesting aspect of this case in the

1 Kuhlman JE, Knowles MC, Fishman EK, Siegelman SS. Premature bullous pulmonary damage in AIDS: CT diagnosis. Radiology 1989; 173: 23-6.

2 Eng RH, Bishburg E, Smith SM. Evidence for destruction of lung tissue during Pneumocystis carinii infection. Arch Intern Med 1987; 147: 746-9.

3 Liu YC, Thomashefski JF Jr, Tomford JW, Green H. Necrotizing Pneumocystis carinii vasculitis associated with lung necrosis and cavitation in a patient with lung necrosis and cavitation in a patient with acquired 113: 494-7.

4 Chow C, Templeton PA, White CS. Lung cysts associated with Pneumocystis carinii pneumonia: radiographic characwith Pneumocystis carinii pneumonia: radiographic characteristics,

5 Sandhu JS, Goodman PC. Pulmonary cysts associated with Pneumocystis carinii pneumonia in patients with AIDS. Radiology 1989; 173: 33-5.
Infectious causes of lung cavities in HIV-positive patients

\section{Common}

- Pneumocystis carinii pneumonia

- tuberculosis

Uncommon

- atypical mycobacteria

- histoplasmosis

- coccidioidomycosis

- cryptococcal pneumonia

- invasive aspergillosis

- Nocardia infections

- Rhodococcus equi infection

- bacterial pneumonia, septic emboli, and lung abscess

remarkable improvement of the lesions as evidenced by chest X-rays and particularly by the CT scan. Although resolution of cavitary infiltrates and pneumatoceles in $P$ carinii pneumonia has been previously described, ${ }^{4-6}$ most of these lesions were relatively small and CT scan, with higher discriminant power than chest X-ray, was not used to ascertain the resolution of the lesions. In fact, the only series that used CT scans to study bullous disease in AIDs did not report the follow-up of the patients. ${ }^{1}$ To our knowledge this is the first report that describes the reversibility of such extensive lesions involving both lungs with the aid of sequential CT scans.

Several mechanisms have been suggested to explain the pathogenesis of the lung cavities in $P$ carinii pneumonia. These include checkvalve bronchiolar obstruction with distal cyst formation, ${ }^{5}$ elastase release from the macrophages with subsequent destruction of the alveolar tissue, ${ }^{2}$ direct or indirect cytotoxic effect of HIV, ${ }^{1}$ and arterial invasion with thrombosis of the vessels, necrosis and cavitation, ${ }^{3}$ among others. However, a destructive process probably did not cause the disease in our patient, as only minimal residual lesions remained in the lung despite the extensive involvement. Aerosolized pentamidine has also been related to pulmonary cysts and pneumothorax, ${ }^{10}$ but our patient never received this drug. Thus, the present case does not support a role of irreversible causes of lung destruction, such as necrosis, in the cavitary lesions observed in $P$ carinii pneumonia. However, the mechanism of these lesions may differ from patient to patient and probably no unifying explanation can account for all cases.

6 DeLorenzo LJ, Huang CT, Maguire GP, Stone DJ. Roentgenographic patterns of Pneumocystis carinii pneumonia in 104 patients with AIDS. Chest 1987; 91: 323-7.

7 Moss S, Carey PB, Hind CRK. Pneumocystis carini pneumonia presenting with pneumomediastinum in an HIV-positive patient. Postgrad Med $\mathcal{F} 1995$; 71: 96-7.

8 Wasserman K, Pothoff G, Kirn E, Fätkenheuer G, Krueger GRF. Chronic Pneumocystis carinii pneumonia in AIDS Chest 1993; 104: 667-72.

9 Goodman PC. The chest film in AIDS. In: Sande MA Volverding PA, eds. The medical management of AIDS. Philadelphia: WB Saunders, 1995; pp 592-613.

10 Jules-Elysée KM, Stover DE, Zaman MB, Bernard EM White DA. Aerosolized pentamidine effect on diagnosis and presentation of Pneumocystis carinii pneumonia. Ann Intern Med 1990; 112: 750-7. 